\title{
Generation of Hyperchaos from the Lü System with a Sinusoidal Perturbation
}

\author{
Philippe Djondiné1, Paul Abena Malobé2 \\ ${ }^{1}$ Department of Physics, Higher Teacher Training College, The University of Ngaoundéré, Bertoua, Cameroon \\ ${ }^{2}$ Department of Physics, Faculty of Sciences, The University of Ngaoundéré, Ngaoundéré, Cameroon \\ Email: pdjondine@yahoo.fr
}

How to cite this paper: Djondiné, P. and Malobé, P.A. (2021) Generation of Hyperchaos from the Lü System with a Sinusoidal Perturbation. Journal of Applied Mathematics and Physics, 9, 1100-1107. https://doi.org/10.4236/jamp.2021.95076

Received: January 27, 2021

Accepted: May 25, 2021

Published: May 28, 2021

Copyright $\odot 2021$ by author(s) and Scientific Research Publishing Inc. This work is licensed under the Creative Commons Attribution International License (CC BY 4.0).

http://creativecommons.org/licenses/by/4.0/

\begin{abstract}
This paper introduces a hyperchaotic system from the Lü system with a sinusoïdal perturbation. This hyperchaotic system has more complex dynamical behaviors, and can generate 2-scroll hyperchaotic attractor and 2-scroll chaotic attractor under different control parameters. Theoretical analyses and simulation are conducted to investigate the dynamical behaviors of the proposed hyperchaotic system by means of Lyapunov exponents, analysis of the bifurcation diagram and phase portraits.
\end{abstract}

\section{Keywords}

Hyper-Chaotic, Bifurcation, Lü System, Lyapunov Exponent

\section{Introduction}

Therefore, much attention has been paid to the investigation of the existence and applications of hyperchaos. It is well known that hyperchaos can only appear in four- or more dimensional systems and is usually generated from a known low-dimensional system [1]-[8] via some control schemes. With the generating mechanisms, some hyperchaotic systems have been discovered as in [9]-[16]. In this letter, we will report a new hyperchaotic system constructed from the Lü system [17] via a sinusoidal perturbation. The Lü system found in 2004 [17] has many interesting properties such as the existence of two-scroll chaotic attractors with only three equilibria and two 2 -scroll chaotic attractors with five equilibria. It is perhaps expected that the Lü system with a sinusoidal perturbation will exhibit more complex dynamical behaviors.

\section{The Lü System}

Lü system is a bridge between Lorenz system and Chen system, and it realizes a 
transition from a system to another system. It is also the simplest chaotic attractor among the unified chaotic system. The Lü system [17] is described as

$$
\left\{\begin{array}{l}
\dot{x}=a(y-x) \\
\dot{y}=-x z+b y \\
\dot{z}=x y-c z
\end{array}\right.
$$

where $\mathrm{x}, \mathrm{y}$, and $\mathrm{z}$ are the state variables, and $\mathrm{a}, \mathrm{b}$, and $\mathrm{c}$ are three system parameters. When $(a, b, c)=(36,20,3)$, system (1) demonstrates a chaotic attractor, shown in Figure 1 and the Lyapunov dimension is 2.0669 .

Hyperchaotic systems, generally, are classified as chaotic systems with more than one positive Lyapunov exponent, this shows that the chaotic dynamics of the systems are expanded in more than one direction and may give rise to more complex attractors. Hyperchaotic system also should satisfy some basic properties:

1) Hyperchaos exists only in higher dimensional systems, i.e. not less than $4 \mathrm{D}$ autonomous system for the continuous time cases.

2) It was suggested that the number of terms in the coupled equations giving rise to instability should be at least two, in which one should be a nonlinear function.

In this study is constructed from (1) a new Lü system with a sinusoidal perturbation on parameter $\mathrm{c}$ in the original Lü system. The new system is described as

$$
\left\{\begin{array}{l}
\dot{x}=a(y-x) \\
\dot{y}=-x z+b y \\
\dot{z}=x y-c(1+\varepsilon \sin \omega t) z
\end{array}\right.
$$

where $\varepsilon$ is the perturbation amplitude, and $\omega$ is the perturbation angular frequency. The addition of the time varying parametric perturbation $a \sin \omega t$ changes the autonomous system (1) to the non-autonomous system (2), which is equivalent to a four-dimensional autonomous system.

This work, we show that the system (2) is hyperchaotic (Figure 2) for the parameter values $a=36, b=20, c=10, \varepsilon=0.43, \omega=23$.

The conditions for achieving hyperchaos are as follows:

1) The minimal dimension of the phase space should be at least four for continuous time systems of integer order.

2) The system has at least two positive Lyapunov exponents, and the sum of all Lyapunov exponents is less than zero.

Using Wolf's algorithm [18], the Lyapunov exponents of the new hyperchaotic system (2) are calculated for $a=36, b=20, c=10, \varepsilon=0.43, \omega=23$ and $X(0)=(0.1,0.1,0.1,0.1)$ for $T=1 \times 10^{4}$ seconds as $L_{1}=1.8357, L_{2}=0.0004565, \quad L_{3}=-21.8628$.

Since the Lyapunov exponents $L_{1}$ and $L_{2}$ are positive, we conclude that the new system (2) is hyperchaotic. Also, by adding all the Lyapunov exponents, we get their sum as $L_{1}+L_{2}+L_{3}=-20.0266435$. This shows that the new hyperchaotic system (2) is dissipative. 

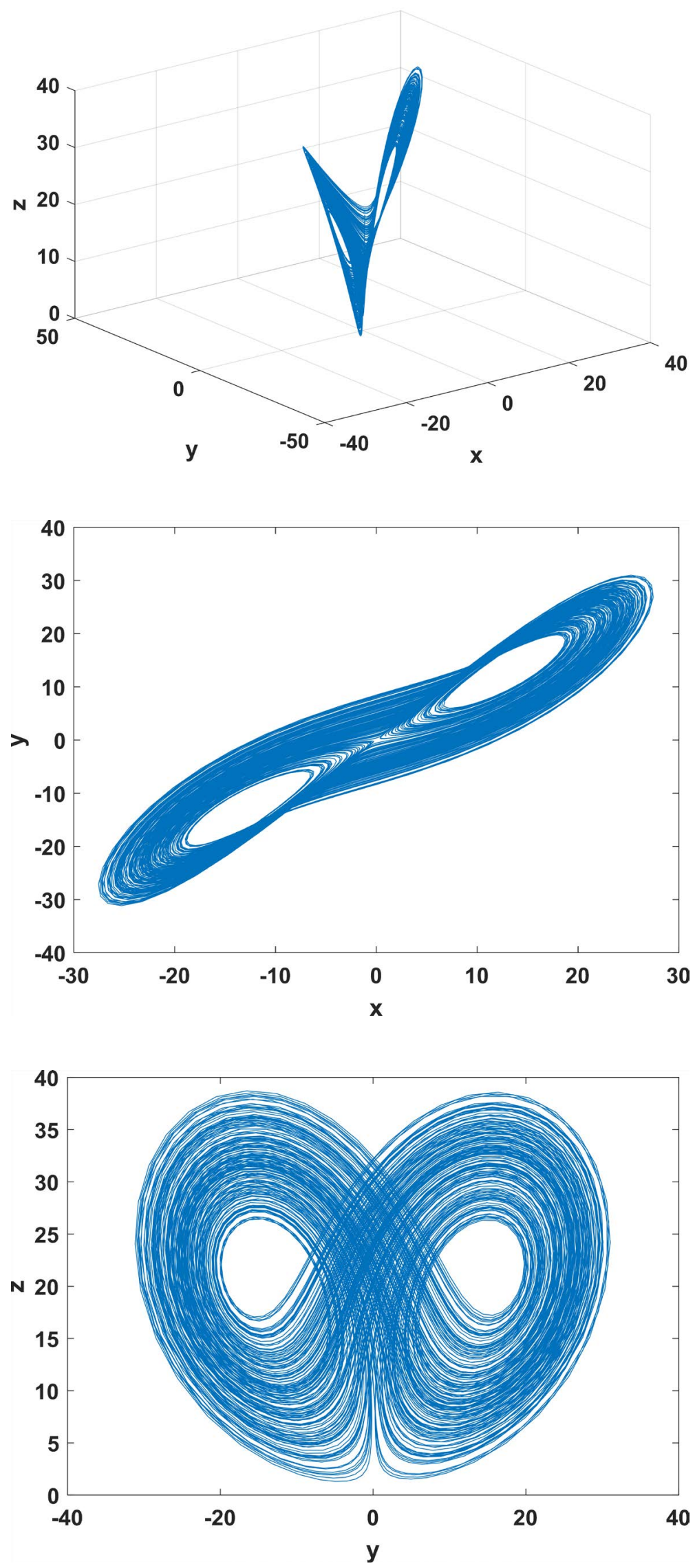


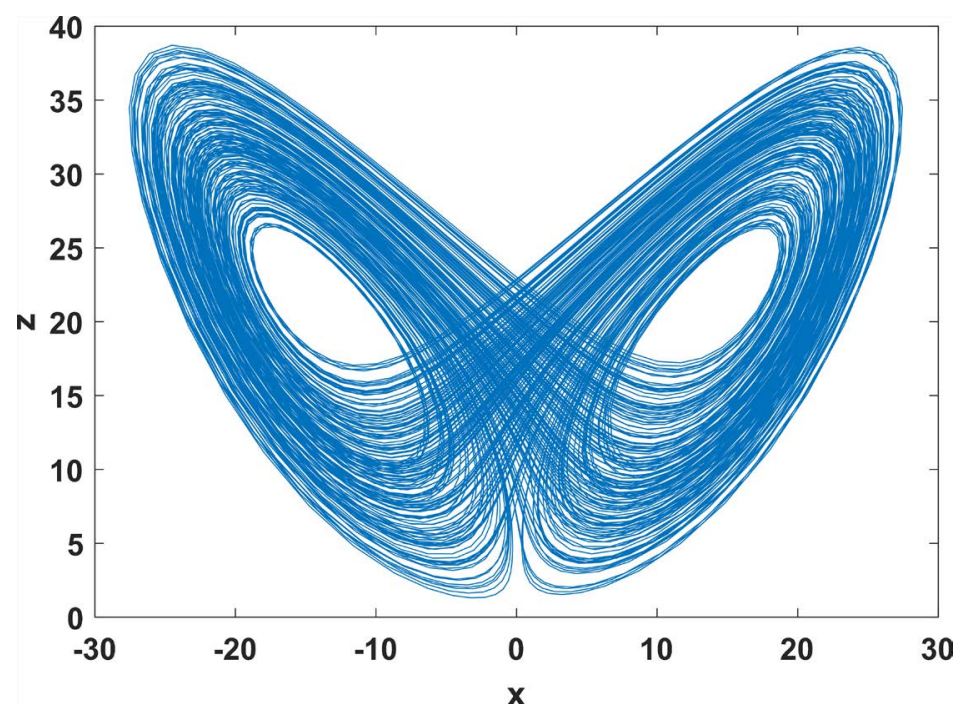

Figure 1. Strange attractors of system (1) with $(a, b, c)=(36,20,3)$.

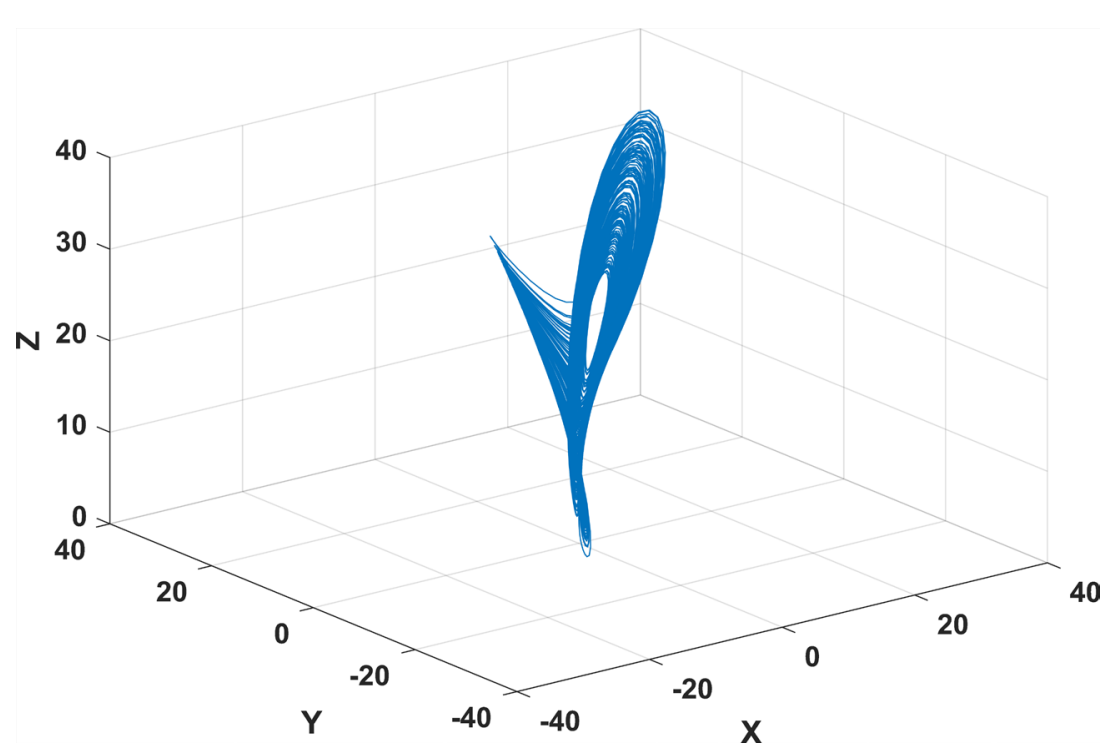

Figure 2. Phase portraits of system (2).

\section{Numerical Simulation}

In this section, we show how the route to hyperchaos is obtained using bifurcation diagram and phase portraits. This new hyperchaotic system is numerically solved using fourth-order Runge-Kutta formula. For each iteration, the time grid is always $\Delta t=2 \times 10^{-6}$ seconds and the computations are made using parameters and variables in extended mode. Bifurcation diagrams are is exploited to define the type of behavior leading to hyperchaos. The Lyapunov exponent is calculated using the method described by Wolf et al. [18]. The spectrum of Lyapunov exponents (Figure 3 ) agrees with bifurcation sequence. In Figure 4, the bifurcation diagram depicts plots of local maxima of the variable $z$ in terms of the control parameter $c$ in the range $1 \leq c \leq 2$. To better clarify that the new hyperchaotic system develop period-doubling scenario, a bifurcation diagram and 


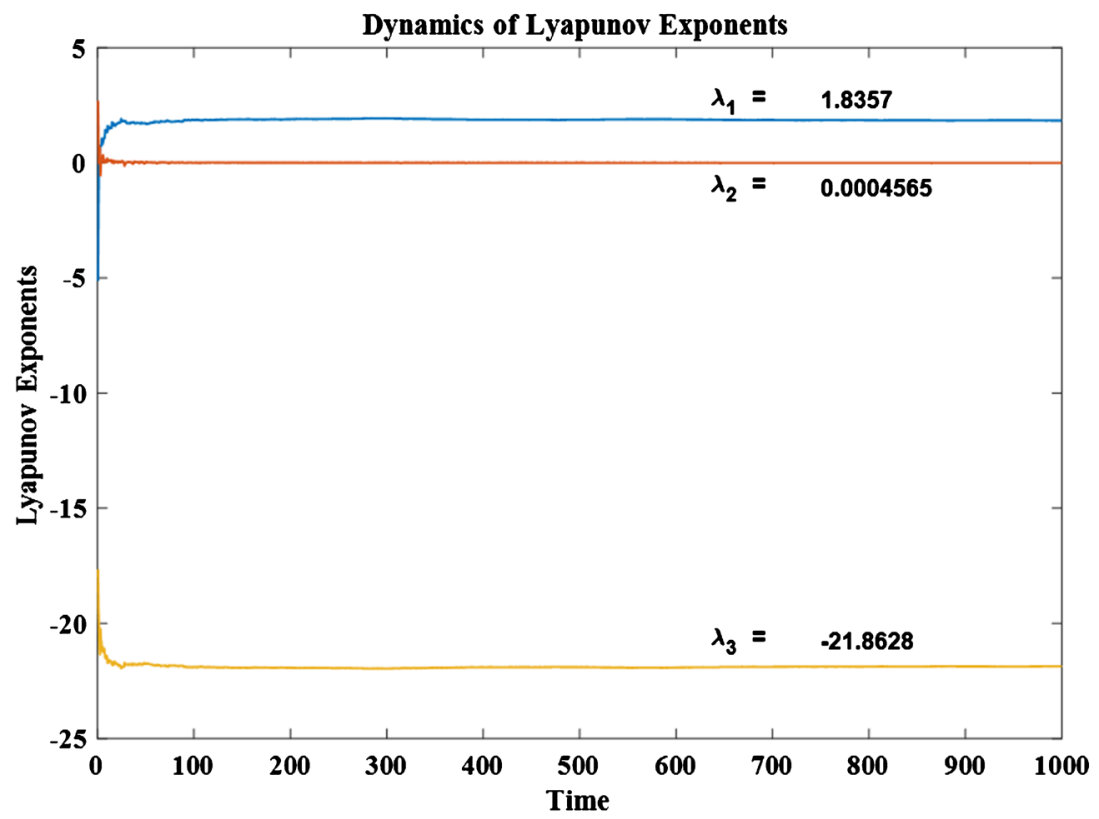

Figure 3. Lyapunov exponents spectrum of system (2).

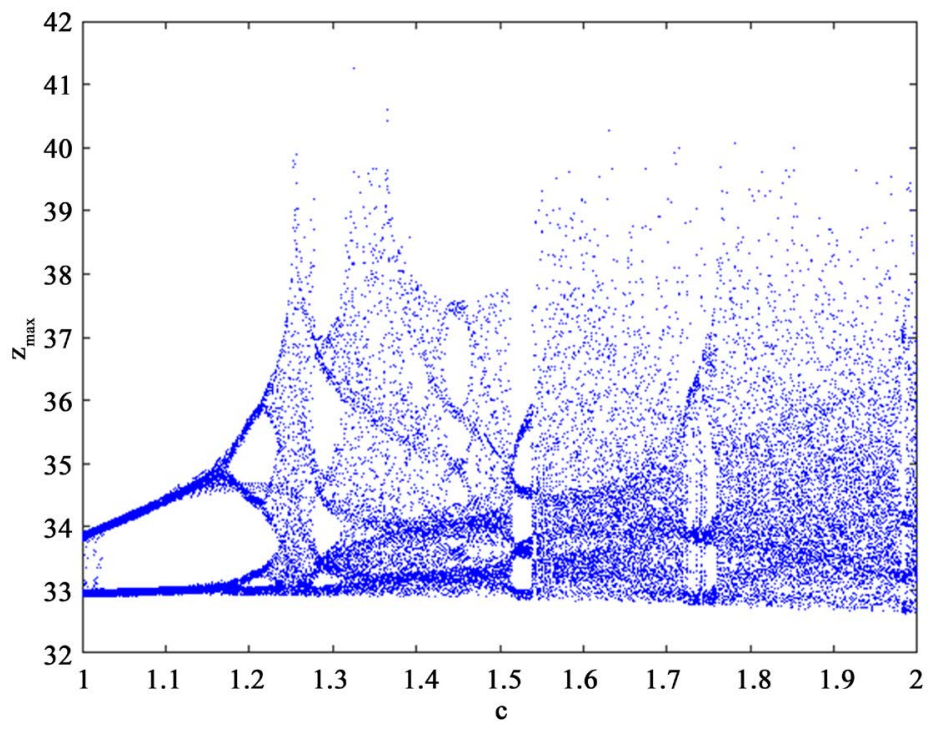

Figure 4. Bifurcation diagram of system (2) for varying c, with $\varepsilon=0.2, \omega=23$.

corresponding Lyapunov exponents are plotted for varying in the range for and with the same initial condition. Figure 5 shows the 2-D phase plots of the new system (2). It is seen that the new system (2) exhibits two-scroll attractor.

\section{Conclusion}

By introducing a sinusoïdal perturbation, this paper presents a Lü system to generate the hyperchaotic behaviors. We discussed dynamic properties such as Lyapunov exponents, phase portraits and bifurcation diagram. The new system has more complex dynamical behaviors, and can generate 2-scroll hyperchaotic attractor in wide parameter ranges. It is very possible that a coexisting intermittent 


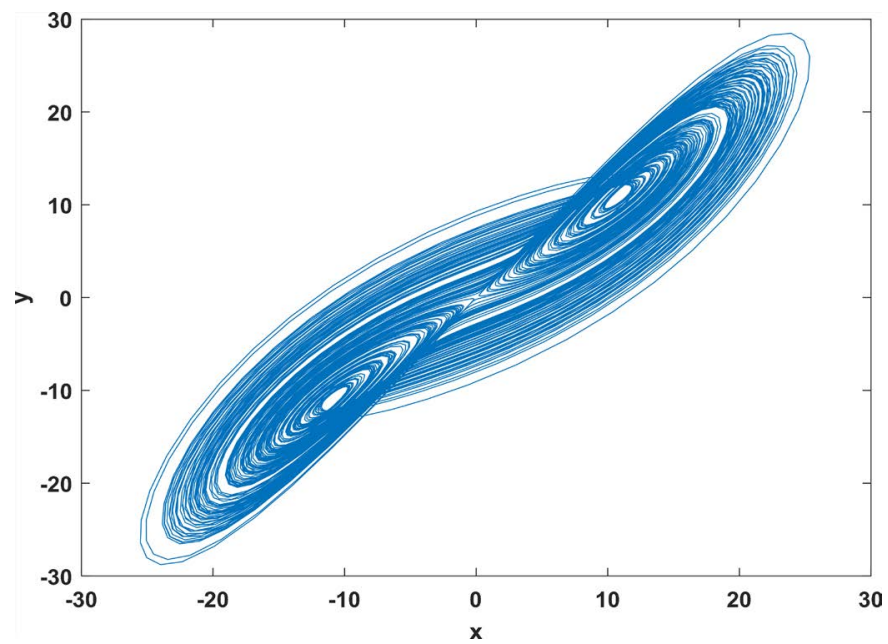

(a)

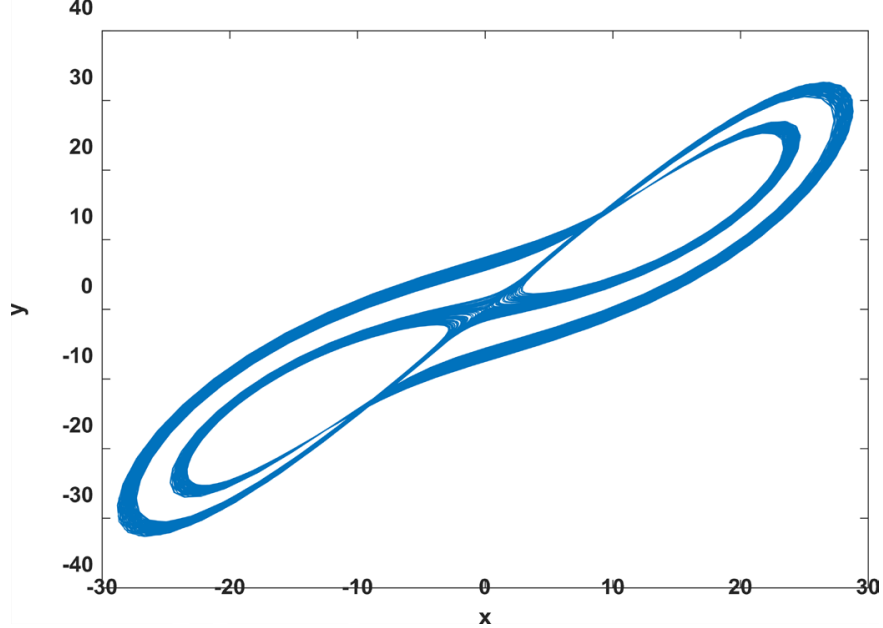

(b)

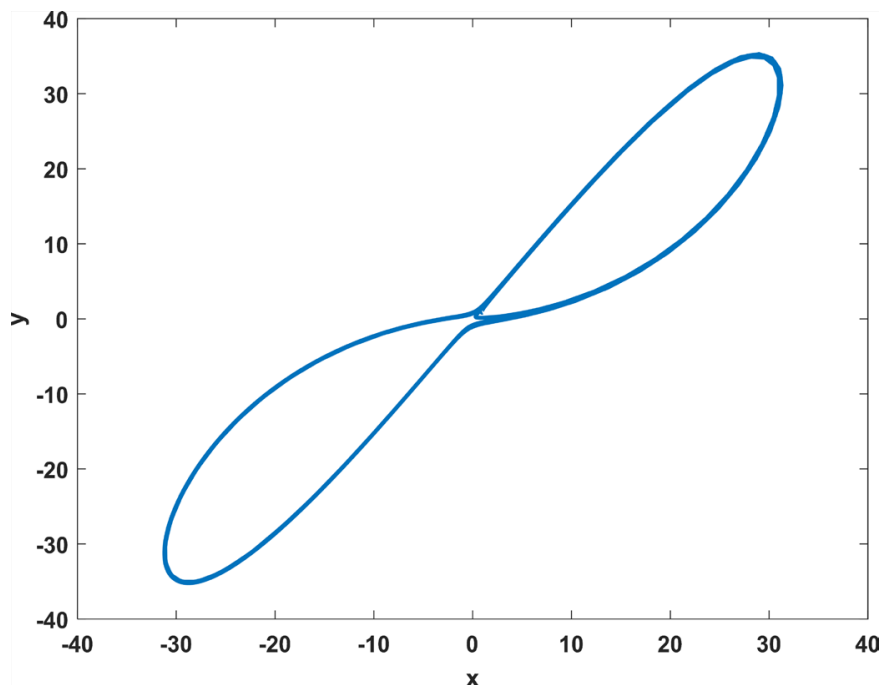

(c)

Figure 5. 2D phase portraits of the hyperchaotic system obtained for some specific values of the control parameter: (a) hyperchaos for $c=10$, (b) torus state for $c=20$ and (c) periodic orbit for $\mathrm{c}=32$. 
chaotic attractor exists in the hyperchaotic system. As the control parameter varies, this proposed system can be periodic, quasi-periodic, chaotic and hyperchaotic, respectively.

\section{Acknowledgements}

The authors would like to thank the journal editor and all organizations that provided data for this research.

\section{Conflicts of Interest}

The authors declare no conflicts of interest regarding the publication of this paper.

\section{References}

[1] Lorenz, E.N. (1963) Deterministic Nonperiodic Flow. Journal of the Atmospheric Sciences, 20, 130-141. https://doi.org/10.1175/1520-0469(1963)020<0130:DNF>2.0.CO;2

[2] Stewart, I. (2002) The Lorenz Attractor Exists. Nature, 406, 948-949. https://doi.org/10.1038/35023206

[3] Rössler, O.E. (1979) An Equations for Hyperchaos. Physics Letter A, 71, 155-169. https://doi.org/10.1016/0375-9601(79)90150-6

[4] Chen, G.R. and Ueta, T. (1999) Yet Another Chaotic Attractor. International Journal of Bifurcation and Chaos, 9, 1465-1469. https://doi.org/10.1142/S0218127499001024

[5] Lü, J. and Chen, G. (2002) A New Chaotic Attractor Coined. International Journal of Bifurcation and Chaos, 12, 659-661. https://doi.org/10.1142/S0218127402004620

[6] Lü, J., Chen, G. and Zhang, S. (2002) Dynamical Analysis of a New Chaotic Attractor. International Journal of Bifurcation and Chaos, 12, 1001-1015. https://doi.org/10.1142/S0218127402004851

[7] Liu, W. and Chen, G. (2003) A New Chaotic System and Its Generation. International Journal of Bifurcation and Chaos, 13, 261-267. https://doi.org/10.1142/S0218127403006509

[8] Liu, C., Liu, T., Liu, K., et al. (2004) A New Chaotic Attractor. Chaos, Solitons \& Fractals, 22, 1031-1038. https://doi.org/10.1016/j.chaos.2004.02.060

[9] Bao, B.-C. and Liu, Z. (2008) A Hyperchaotic Attractor Coined from Chaotic Lü System. Chinese Physics Letters, 25, 2396-2399.

https://doi.org/10.1088/0256-307X/25/7/018

[10] Chen, A., Lu, J., Lü, J. and Yu, S. (2006) Generating Hyperchaotic Lü Attractor via State Feedback Control. Physics A, 364, 103-110. https://doi.org/10.1016/j.physa.2005.09.039

[11] Chen, Z. Yang, Y., Qi, G. and Yuan, Z. (2007) A Novel Hyperchaos System Only with One Equilibrium. Physics Letters A, 360, 696-701. https://doi.org/10.1016/j.physleta.2006.08.085

[12] Jia, Q. (2007) Hyperchaos Generated from the Lorenz Chaotic System and Its Control. Physics A, 366, 217-222. https://doi.org/10.1016/j.physleta.2007.02.024

[13] Li, Y.X., Wallace Tang, K.S. and Chen, G.R. (2005) Generating Hyperchaos via State Feedback Control. International Journal of Bifurcation and Chaos in Applied Sciences 
and Engineering, 15, 3367-3375. https://doi.org/10.1142/S0218127405013988

[14] Li, Y.X., Wallace Tang, K.S. and Chen, G.R. (2005) Hyperchaos Evolved from the Generalized Lorenz Equation. International Journal of Circuit Theory and Applications, 33, 235-251. https://doi.org/10.1002/cta.318

[15] Wang, F.-Q. and Liu, C.-X. (2006) Hyperchaos Evolved from the Liu Chaotic System. Chinese Physics, 15, 963-968. https://doi.org/10.1088/1009-1963/15/5/016

[16] Wang, G., Liu, J. and Zheng, X. (2007) Analysis and Implementation of a New Hyperchaotic System. Chinese Physics B, 16, 2278-2284.

https://doi.org/10.1088/1009-1963/16/8/020

[17] Lü, J., Chen, G. and Cheng, D. (2004) A New Chaotic System and Beyond: The Generalized Lorenz-Like System. International Journal of Bifurcation and Chaos, 14, 1507-1537. https://doi.org/10.1142/S021812740401014X

[18] Wolf, A., Swift, J.B., Swinney, H.L. and Vastano, J.A. (1985) Determining Lyapunov Exponents from a Time Series. Physica D, 16, 285-317.

https://doi.org/10.1016/0167-2789(85)90011-9 